\title{
Alexis de Tocqueville in China: Spiegeldebatten über Reformbedarf und Revolutionsgefahr
}

Was haben die Phase nach der Französischen Revolution und die chinesische Gegenwart des 21. Jahrhunderts gemeinsam? Auf den ersten Blick scheint es sich um zwei Systeme und Gesellschaftsmodelle zu handeln, die konträrer nicht sein könnten. Der Ruf nach Freiheit, Demokratie und Menschenrechten, welcher mit der Französischen Revolution von 1789 assoziiert wird, steht für ein pluralistisches Modell, die Emanzipation des Bürgertums und den Umsturz der alten Ordnung. Umso erstaunlicher, dass Tocquevilles Studie Der alte Staat und die Revolution ${ }^{1}$ von der chinesischen politischen Führung nicht als Aufruf zum Umsturz des chinesischen EinPartei-Systems, sondern als Orientierung und Denkanstoß für mögliche Reformen in der Volksrepublik (VR) China aufgegriffen worden ist. ${ }^{2}$ Die Leseempfehlung (beziehungsweise Leseanweisung), durch welche die chinesische Übersetzung von Tocqueville zu einem Schlüsseltext der Spitzen der chinesischen Kaderelite avanciert ist, soll von niemand Geringerem als Wang Qishan stammen, Mitglied im Ständigen Ausschuss des Politbüros ${ }^{3}$ und mit der Bekämpfung der immer weiter um sich greifenden Korruption beauftragt. Neben Tocqueville legte Wang Qishan seinen Politbürokollegen zudem die chinesische Übersetzung der von Michael Sandel herausgegebenen Textsammlung Justice 4 sowie die Verfilmung des Politromans Das Kartenhaus (engl. Originaltitel: House of cards) von Michael Dobbs ${ }^{5}$ nahe. Letzterer thematisiert fiktional die Korruption und Machtspiele von Politikern im Wahlkampf und die Instrumentalisierung der Medien. Der Schauplatz ist Großbritannien während der frühen 1990er Jahre. Drei nichtchinesische Bücher dienen dem Politbüro als Orientierungsmodelle. Nur ein chinesisches Buch wurde von Wang benannt: Der Minister der Qing-Dynastie (Da Qing xiangguo) von Wang Yuewen, ${ }^{6}$ ein Roman über den kaiserlichen Minister Chen Tingjing, seinen (vorbildli-

1 Toqueville 2013 [1856].

2 Liao 2013; vgl. auch das (unveröffentlichte) Vortragsmanuskript von Helwig SchmidtGlintzer zum Thema »Herausforderung und Response«(Bonn, 4. November 2013).

3 Der Ständige Ausschuss des Polibüros gilt als oberstes (kollektives) Führungsgremium und Schaltzentrale der chinesischen Politik.

4 Sandel 2007.

5 Dobbs 1989.

6 Wang 2010. 
chen) Führungsstil und seine unermüdlichen Bemühungen, eine moderne Staatsverwaltung aufzubauen. ${ }^{7}$

Die Auseinandersetzung mit westlichen Romanen und staatsphilosophischen Schriften bedeutet allerdings nicht, dass eine Restrukturierung des chinesischen Systems nun nach dem Muster demokratisch-kapitalistischer Staaten und Gesellschaftssysteme erfolgen soll. Vielmehr werden Systemunterschiede hinter der Frage, wie eine moderne, effektive und transparente Verwaltung aufgebaut werden könnte, zurückgestellt. Die Beschäftigung mit den oben genannten Texten steht folglich in der Tradition der seit Jahren in der VR China laufenden Lern- und Adaptionsprozesse, ${ }^{8}$ welche auf der Beobachtung und Analyse von Entwicklungsprozessen und staatstheoretischen Grundmustern in der chinesischen Geschichte, aber auch solchen in anderen politischen Systemen beruhen. À la Hegel geht es darum, aus der Geschichte zu lernen. ${ }^{9}$

Kontinuität, Stabilität und die Persistenz des Ein-Partei-Systems rangieren auf der Prioritätenliste der chinesischen politischen Führung weiterhin an oberster Stelle. China wird regiert von einer kommunistischen, nach leninistischen Organisationsprinzipien strukturierten Kaderpartei. Grundprinzip ist weiterhin das Konzept des demokratischen Zentralismus. Die gegenwärtige Strategie des Machterhalts beruht auf einer Kombination aus institutioneller Einbindung und Kooptation. Die Partei hat ihren Vertretungsanspruch offiziell ausgebaut und ist damit zu einer Massenpartei mutiert, die neben Arbeitern, Bauern und Soldaten nun die Interessen der ökonomischen und intellektuellen Eliten mitvertritt. Während sich das Profil gewandelt hat, sind jedoch die ideologischen Grundstrukturen nicht aufgegeben, sondern lediglich erweitert worden. Offiziell hält die Partei weiterhin an den Ideen des Marxismus(-Leninismus) und ihrer Sinisierung fest. Um ihr Machtmonopol abzusichern, beruft sich die Partei auf ihre Erfolge, also die "Output-Dimension " ihrer Legitimität: Unter der Führung durch die Kommunistische Partei Chinas (KPCh) hat die VR China ein lange Zeit zweistelliges Wirtschaftswachstum erzielt und ist nun auf dem Sprung, die USA als weltweit führende Volkswirtschaft abzulösen - ein Ziel, das sie inzwischen laut einer Studie der Weltbank - von ihrer kom-

7 Diese Liste wurde 2013 auf diversen chinesischen Mikroblogs gepostet und diskutiert. Abgedruckt findet sie sich zudem in Jun Gong Wenhua (Military Industry Culture) 1 (2014).

$8 \mathrm{Zu}$ den chinesischen Lern- und Adaptionsprozessen vgl. insbesondere Shambaugh 2008. Die Fähigkeit autokratischer Systeme, sich an veränderte Umfeldstrukturen flexibel anzupassen und damit ihre Persistenz zu sichern, thematisiert Nathan 2003.

9 Auch die früheren Führungseliten der VR China suchten Inspiration in der europäischen Staatstheorie, nicht zuletzt den Klassikern der griechischen und römischen politischen Philosophie. Exemplarisch verdeutlicht dies auch die Rezeption der Schriften von Carl Schmitt und Leo Strauss (hierzu Lilla 2011). Rezipiert wird selektiv: Die konkurrierenden Faktionen innerhalb der Partei wie auch die verschiedenen intellektuellen Gruppierungen, die weitaus diverser sind, als die pauschalisierenden Oberbegriffe Neoliberale versus Neue Linke vermuten lassen, greifen eklektisch Ideen oder Fragmente auf, welche ihren jeweiligen Standpunkt untermauern. 
parativen Wirtschaftsleistung her gesehen - auch erreicht hat. ${ }^{10}$ Doch neben der Fixierung der Rahmenbedingungen für ein stabiles positives Wirtschaftswachstum beansprucht die Partei zudem, die territoriale Einheit und nationale Souveränität der VR China zu garantieren und die nationalen Interessen gegenüber dem Ausland zu behaupten.

Infolge der Reform- und Öffnungspolitik (eingeleitet durch die Beschlüsse des 3. Plenums des 11. ZK 1978) hat jedoch eine Fragmentierung der chinesischen Gesellschaft eingesetzt, die sich gegenwärtig in Form sozio-ökonomischer Spannungen zwischen den verschiedenen Einkommensgruppen entlädt und letztendlich das Überleben des politischen Regimes in seiner Gesamtheit infrage stellt. ${ }^{11}$ Wie können derartige Ungleichheiten und Entwicklungsdisparitäten in einem offiziell weiterhin sozialistischen System begründet beziehungsweise relativiert werden? ${ }^{12}$ Getrieben von der Vorstellung, dass zu große Spannungen zu einem Untergang des Systems führen könnten, arbeitet die chinesische Führung unter Hochdruck an Plänen, wie ein nachhaltigeres und sozial verträglicheres Wirtschaftswachstum erzielt werden könnte. Insbesondere die Lage der sozial benachteiligten Gesellschaftsgruppen - der Wanderarbeiter und der Landbevölkerung allgemein - sorgt für Kopfzerbrechen. Das Problem besteht darin, dass neue redistributive Maßnahmen, die nur durch die Erhöhung von Steuern zu realisieren wären, zu Spannungen und Konflikten zwischen dem Partei-Staat und den ökonomischen Eliten führen könnten. Diese Gruppe, der in Transformationstheorien zugeschrieben wird, politische Rechte einzufordern und über kurz oder lang den Zusammenbruch des Systems zu bewirken, hat sich wider Erwarten als eine der tragenden Stützen des politischen Systems herausgestellt. Es besteht eine Art Symbiose zwischen dem Partei-Staat und den kapitalistischen Akteuren, auch als »rote Kapitalisten « bezeichnet. ${ }^{13}$ Seit 2002 können diese auch in die KPCh aufgenommen werden. Sollte der chinesische Partei-Staat diese Gruppe stärker in die Pflicht nehmen und zur Kasse bitten, dürfte das fragile politisch-ökonomische Gleichgewicht zwischen Partei und Unternehmern massiv ins Schwanken geraten. Das Szenario einer potenziellen Krisenerschütterung des Systems - die sowohl dann eintreten könnte, wenn die sozio-ökonomischen Spannungen innerhalb der Gesellschaft sich weiter zuspitzen, als auch dann zu erwarten wäre, wenn die Sonderrechte bestimmter Elitengruppen beschnitten würden - führt thematisch weiter zur Frage nach dem Verhältnis von Reform und Revolution.

10 Siehe Spiegel online vom 30. April 2014. www.spiegel.de/wirtschaft/soziales/china-verd raengt-usa-als-groesste-volkswirtschaft-der-welt-a-966981.html

(Zugriff vom 28.07.2014).

11 Tong, Lei 2010.

12 Bereits in den 1990er (!) Jahren hatte Dieter Senghaas auf die zu erwartenden negativen ökologischen und sozio-ökonomischen Begleiterscheinungen der Reformbeschlüsse von 1978 hingewiesen (Senghaas 1996, S. 85-88). Die gegenwärtigen Probleme sind somit langfristig absehbar gewesen - doch zu Gunsten einer blinden Fokussierung auf Modernisierung und Wohlstandssteigerung zunächst von der chinesischen Regierung in Kauf genommen worden.

$13 \mathrm{Zu}$ den »roten Kapitalisten « und ihrer Rolle im politischen System der VR China vgl. Dickson 2003. 
Reformstau und drohende Systemkrisen dominieren die Bedrohungsszenarien, die innerhalb der politischen Führung diskutiert werden. Die weiterhin zentrale Herausforderung der chinesischen Parteiführung ist, wie die Zusammenstellung der obigen empfohlenen Pflichtlektüre für Kader verdeutlicht, die Bekämpfung von Amtsmissbrauch und Korruption. Ein korruptes System gilt nicht nur als ineffizient, sondern birgt auch die Gefahr eines unerwartet auftretenden Systemkollapses. In der chinesischen Geschichte erfolgte die Ablösung einer Dynastie durch einen neuen Herrscher mit der in den jeweiligen Dynastiegeschichten verankerten Erklärung, dass mit der Errichtung der neuen Dynastie die Ordnung wiederhergestellt und dem korrupten, dekadenten Treiben der Vorgängerdynastie ein Ende gesetzt worden sei. Die Unterbindung der Korruption ist folglich für das politische System (über)lebensnotwendig. Machtwillkür und Korruption lokaler Kader haben in den letzten Jahren wiederholt zu Massenprotesten der betroffenen Bevölkerung geführt - oft genug hat in solchen Fällen die Zentralregierung durchaus zu Gunsten der Protestierenden interveniert und Untersuchungsverfahren eingeleitet. ${ }^{14}$ Dennoch - die fortbestehende Korruption und individuelles Fehlverhalten der Parteikader tragen weiterhin dazu bei, dass das allgemeine Vertrauen der Bevölkerung in den chinesischen ParteiStaat sinkt. ${ }^{15}$ Neben dieser potenziellen Legitimitätskrise auf lokaler Systemebene könnte dem System eine Steuerungs- und Durchsetzbarkeitskrise drohen, sofern es nicht gelingen sollte, die bürokratischen Strukturen zu modernisieren und weitere Prüf- und Ausgleichs-Mechanismen aufzubauen. Die Einrichtung entsprechender Kontroll- und Beschwerdestellen, die eine Ebene oberhalb der durch die Bevölkerung angeklagten lokalen Partei- und Regierungsorgane angesiedelt sein sollen, hat die Kommunistische Partei der VR China auf ihrem 3. Plenum im November 2013 beschlossen. ${ }^{16}$ Rechtsstaatlichkeit - worunter die Herrschaft durch Recht (rule by law), jedoch nicht die Herrschaft des Rechts (rule of law) verstanden wird - und soziale Gerechtigkeit sind die Eckpfeiler dieser Bemühungen um eine Legitimierung des Systems.

$\mathrm{Zu}$ beobachten ist die Suche nach »neuen « Orientierungsmodellen insbesondere in Umbruchphasen und Krisenzeiten. So reiste kurz vor dem Zusammenbruch des chinesischen Kaiserreichs und der Ausrufung der Republik China (1912) eine chinesische Delegation durch Europa mit dem Auftrag, die dortigen Verfassungen und Parteistrukturen zu analysieren und hieraus Empfehlungen für den Aufbau einer konstitutionellen Monarchie in China abzuleiten. Der frühen maoistischen VR China wiederum diente die Sowjetunion als Vorbild und Ideengeber (auch wenn sich dies in den 1950er Jahren bald schon ändern sollte und mit den Mao-Zedong-

14 Noesselt 2014, S. 142-145.

15 Die Berichte der New York Times zu Korruption einzelner Mitglieder in den Reihen des Ständigen Ausschusses des Politbüros wurden im chinesischen Internet zensiert, was die Sprengkraft, die dem Thema Korruption mit Blick auf das Überleben des Systems zugeschrieben wird, verdeutlicht.

16 Eine deutschsprachige Version des Communiqué des 3. Plenums des 18. ZK findet sich unter: http://german.china.org.cn/china/2014-01/15/content_31202962.htm (Zugriff vom 28.07.2014). 
Ideen eine Sinisierung des sozialistischen Modells vollzogen wurde). Nach 1978 wiederum waren es die »westlichen « Systeme, welche Pate für die partielle Restrukturierung des chinesischen Wirtschaftssystems standen. Anleihen werden, wie hieraus deutlich wird, nicht notwendigerweise aus den Erfahrungen und Strukturen anderer sozialistischer Staaten abgeleitet, sondern eher im Gegenteil: Die VR China grenzt sich beispielsweise entschieden von der extremen Version des Sozialismus unter Kim Jong Un ab und sieht allenfalls in liberal-reformorientierten Sozialismen (zum Beispiel Kuba unter Raúl Castro) mögliche Anknüpfungspunkte für eine weitere Ausdifferenzierung des chinesischen Sozialismus. ${ }^{17}$

Aus dieser Einbettung der Studie Der alte Staat und die Revolution in ein ganzes Bündel von Orientierungsvorlagen wird deutlich, dass Tocqueville nicht unter der Fragestellung Demokratisierung und Pluralisierung, sondern vor dem Hintergrund einer potenziellen Destabilisierung des Systems und der möglichen Gefahr eines revolutionären Aufbegehrens der Verlierer der Wirtschaftsreformen gegen die Herrschaft der Kommunistischen Partei diskutiert wird. Während vier der fünf von Wang Qishan als Schlüsselwerke benannten Studien und fiktionalen Darstellungen sich mit der Output-Seite der Legitimität auseinandersetzen, steht Tocquevilles Studie für eine sehr viel abstraktere Reflektion zum Zusammenspiel von Reform und Revolution. So stellt er nicht die Frage nach dem idealtypischen Staatsaufbau und einer effektiven Verwaltung in den Mittelpunkt seines Forschungsinteresses. Vielmehr untersucht er die Gründe für den Ausbruch der Revolution und unternimmt einen Vergleich zwischen den Systemstrukturen vor und nach 1789. Von allen oben genannten Texten ist Tocquevilles damit derjenige, welcher für die Überlegungen zu möglichen Formen einer langfristigen Ausgestaltung der Beziehungen zwischen Regierung und Bevölkerung am meisten bietet und auch als Analyseraster für die sozio-ökonomische, sozio-politische Gegenwart der VR China herangezogen werden dürfte. Das revolutionäre und post-revolutionäre Frankreich wird damit als Spiegelbild und Lehrstück für die gegenwärtige Lage in China und die Suche nach einem Ausweg aus der Krise gesehen. ${ }^{18}$

Die in China seit $2012^{19}$ laufende Tocqueville-Debatte identifiziert zwei Hauptaussagen zur Französischen Revolution. Die Revolution, so die erste Schlüsselthese Tocquevilles, erfolgte nicht in einer Phase der absoluten Armut und politischen Diktatur, sondern zu einem Zeitpunkt der relativen ökonomischen Prosperität und beginnender politischer Reformen. ${ }^{20}$ Diese These, sofern sie denn abstrakt auf die Lage in China übertragbar ist, hat weitreichende Konsequenzen für die Weichenstellung in der chinesischen Politik: Ökonomisches Wachstum ist spätestens seit

17 Für einen Vergleich verschiedener Spielarten des Sozialismus und der VR China siehe CASS Institut für Marxismus-Studien 2011; Jiang 2010.

18 Zu diesem Punkt und der Überinterpretation von Tocqueville in der gegenwärtigen chinesischen Debatte vgl. Hu 2013.

19 Also seit dem Beginn der Machtübertragung an die neue Führungselite. Auch vor 2012 wurde Tocqueville in China diskutiert, allerdings zumeist unter abstrakten historischen Fragestellungen, die auf den europäischen Raum beschränkt blieben.

20 Zhu 2013, S. 28. 
1978 ein Schlüsselbegriff in der Legitimationsstrategie der Kommunistischen Partei Chinas. Trotz zeitweilig rückläufiger Zuwachsraten in Jahren der Rezession verzeichnet die VR China dennoch weiterhin Wirtschaftswachstum (derzeit offiziell 7 Prozent). Zudem ist die absolute Armut in China seit Beginn der Reform- und Öffnungspolitik (1978) insgesamt deutlich zurückgegangen, wenngleich weiterhin extreme Entwicklungsdisparitäten zwischen den reichen Küstenprovinzen und den (noch) unterentwickelten Westregionen und zwischen Stadt und Land fortbestehen. Insgesamt aber ist das Durchschnittseinkommen in China seit 1978 deutlich gestiegen, die Bevölkerung hat von der Wirtschaftstransformation (trotz all ihrer negativen Externalitäten) profitiert. In Anlehnung an Tocqueville hätte die VR China damit eine Stufe erreicht, auf der verstärkt mit Protesten und Unruhen zu rechnen wäre.

Die zweite These, die in der chinesischen Lesart aus Tocquevilles Werk herausgefiltert wird, besagt, dass die Revolutionäre, sobald sie denn einmal an die Macht gekommen seien, eben jene Praktiken an den Tag legen würden, die sie zuvor mit Blick auf die alte Herrscherklasse kritisiert hätten. Diesem Eindruck versucht die chinesische Führung entgegenzutreten, indem sie offiziell auf den Aufbau einer modernen, dienstleistungsorientierten und transparenten Verwaltung hinarbeitet. Mit einer breit angelegten Anti-Korruptionskampagne, die unter dem Slogan »Tiger und Fliegen töten « steht, ${ }^{21}$ versucht sie, Missstände zu unterbinden und nicht nur symbolisch das Vertrauen der Bevölkerung zurückzugewinnen. Meldungen der internationalen Presse über die Korruption von chinesischen Spitzenkadern und den Transfer ihres Vermögens in internationale Steueroasen, die trotz Internet- und Medienzensur von Teilen der chinesischen Bevölkerung durchaus wahrgenommen wurden, sind im Sinne dieses Bestrebens der Partei natürlich unerwünscht. Allerdings ist zu bedenken, dass es nichtsdestotrotz eine große Sehnsucht nach Reformschritten in Politik, Wirtschaft und auch im Bereich des administrativen Systems gibt und hohe Erwartungen an die seit 2012/2013 im Amt befindliche Führung gestellt werden - diese genießt gegenwärtig durchaus einen Vertrauensvorschuss.

Ein Artikel der chinesischen Ausgabe der Financial Times verbindet die Tocqueville-Debatte mit den chinesischen Demonstrationen des Jahres 1989. Mit der Niederschlagung dieser Bewegung, so der Artikel, sei die (maoistische) Idee der permanenten Revolution durch den Leitgedanken der Aufrechterhaltung des Systems abgelöst worden. Grundsätzlich hätten die Revolutionen seit 1911 nicht zu Freiheit, sondern zu neuen repressiven Strukturen geführt. ${ }^{22}$ Die an Tocqueville angelehnte Einschätzung der post-revolutionären Strukturen bringt eine starke Desillusionierung und Resignation zum Ausdruck. Denn zwar hat die Revolution von 1949 in China dem Anspruch nach ein Ende des »semi-feudalen, semi-kolonialen « Systems eingeleitet, die neuen Strukturen der Gegenwart sind jedoch noch weit entfernt von den ursprünglichen Zielvorgaben des Sozialismus. Der »Abschied von der Revolu-

21 Xinhua 2013.

22 Vgl. den Artikel » Tuokewei'er qu nar le? «(Wo ist Tocqueville?), in Financial Times vom 16. Januar 2014 (Chinese Edition). www.ftchinese.com/story/001054422? page =1 (Zugriff vom 20.05.2014). 
tion " (gaobie geming) zieht sich als Motiv durch die gegenwärtigen politischen Debatten der VR China, ein Handlungsmotiv, das auch zu einem Risiko des Systems werden könnte, wenn mögliche Kurskorrekturen ungeplante grundstürzende Gegeneffekte mit sich bringen sollten.

»Ich wußte, daß zwar eine große Revolution die Freiheit in einem Land begründen kann, aber mehrere aufeinanderfolgende Revolutionen jede geordnete Freiheit für lange Zeit unmöglich machen " ${ }^{23}$ so heißt es bei Tocqueville. Genau dieses Bewusstsein determiniert auch die parteiinternen Überlegungen zu Reform und Revolution. Eine Revolution, so der Grundgedanke, führt zu einer Ablösung der bestehenden Strukturen und einem kompletten Neuaufbau des Systems. Zwischen dem Abschied von der alten Ordnung und der Stabilisierung einer neuen aber liegen erwartungsgemäß Jahre des Chaos (luan), welche Chinas bisherige Entwicklungen um Jahre zurückwerfen könnten. Eine Neuauflage der Kulturrevolution - die noch längst nicht aufgearbeitet, sondern weiterhin im kollektiven Gedächtnis als die »zehn finsteren Jahre « (1966-1976) eingeschrieben ist - soll um (fast) jeden Preis verhindert werden. Allerdings gewinnen neo-maoistische Ideen gerade vor dem Hintergrund wachsender Entwicklungsdisparitäten und sich zunehmend formierender sozioökonomischer Klassenbildungen - in einem, wohlgemerkt, sozialistischen (!) System - durchaus an Zuspruch. ${ }^{24}$ Ein turbokapitalistischer, neoliberaler Kurs, der in den 1980er Jahren zunächst Wohlstandssteigerung für alle versprach, lässt sich gegenwärtig, so der Konsens in der Partei, nicht aufrechterhalten. Denn die negativen Begleiterscheinungen der Wirtschaftsreformen - Wachstumskrise und das Aufkommen massiver Arbeiterproteste - verstärken den Ruf nach Reformen (oder, so die Forderung der Neuen Linken, nach einer Rückkehr zu maoistischen Rezepten). Diesem will die neue Parteiführung unter Xi Jinping und Li Keqiang mit dem Communiqué des 3. Plenums des 18. Zentralkomitees aus dem November 2013 symbolisch nachkommen, wenn auch nur in kleinen Schritten. So soll das soziale Sicherungssystem aus den Überschüssen der Staatsunternehmen finanziert werden. Das Communiqué des 3. Plenums sieht vor, dass diese in Zukunft 30 Prozent ihrer erwirtschafteten Gewinne abzuführen haben. ${ }^{25}$

Parallel hierzu soll die Bedeutung des Marktes gestärkt werden und ein partieller Rückzug des Staates aus der Wirtschaftsführung erfolgen. ${ }^{26}$ Der, Markt, so heißt es nun, solle »eine entscheidende Rolle bei der Verteilung der Ressourcen " spielen. ${ }^{27}$ Damit löst sich die Parteiführung mehr und mehr von dem alten Bild des »Vogels

23 Tocqueville 1954, S. 12 f.

24 Zwei Gruppen konkurrieren in China um die Definition des »korrekten « Wirtschaftsmodells. Während die Neuen Linken für mehr redistributive Maßnahmen plädieren, treten die Anhänger des Neoliberalismus für die Freiheit und die Selbststeuerungskräfte des kapitalistischen Marktsystems ein.

25 Communiqué des 3. Plenums des 18. ZK 2013. http://german.china.org.cn/china/ 2014-01/15/content_31202962.htm (Zugriff vom 10.01.2014).

$26 \mathrm{Zu}$ Chinas gegenwärtiger Wirtschaftskurskorrektur siehe auch Erber 2014.

27 Communiqué des 3. Plenums des 18. ZK 2013. http://german.china.org.cn/china/ 2014-01/15/content_31202962.htm (Zugriff vom 10.01.2014). 
im Käfig «, das im Zuge der Beschlüsse über Reform und Öffnung des 3. Plenums des 11. ZK (1978) geprägt worden war. Der Markt, hier in der Rolle des Vogels, sollte sich (zunächst begrenzt auf die Sonderwirtschaftszonen) frei entfalten, jedoch nie den Käfig der sozialistischen Planwirtschaft verlassen dürfen. Gewiss gilt dieses Bild schon lange als anachronistisch. Während der ganzen Jahre der Reformen wurde mit einer radikalen Trennung zwischen Politik und Wirtschaft operiert (= wirtschaftliche Liberalisierung ohne politische Pluralisierung), nun sind mit dem Communiqué des 3. Plenums von 2013 komplementäre, synchrone Reformen in den fünf Teilbereichen Wirtschaft, Politik (primär: administratives System), Kultur, Gesellschaft, Ökologie vorgesehen.

Ein zweiter Schlüsselbegriff, um den sich die chinesische Debatte rankt, ist das Konzept der demokratischen Freiheit. Folgt auf das Ende der "alten " Herrschaft in der post-revolutionären Phase die Errichtung einer demokratisch-freiheitlichen Ordnung? Oder aber eher eine demokratisch-zentralistische Ordnung? ${ }^{28}$ Chu Jianguo von der Universität Wuhan reduziert die Kernaussage Tocquevilles auf das Paradoxon, wie eine Revolution, die auf die Erringung von Freiheitsrechten abzielt, in der ersten Phase danach genau den gegenteiligen Effekt haben konnte. Er konstatiert, dass die Hauptgefahr für die Freiheit in der Zentralisierung von Macht und der Korrumpierung eben dieses allmächtigen Zentrums bestehe. ${ }^{29}$

Dies steht in kausaler Verbindung zu Betrachtungen über Zentralisierung versus Dezentralisierung, also zu den Machtrelationen zwischen Zentralstaat und Provinzen. Der Zentralismus, so stellt Tocqueville fest, ist kein Produkt der Revolution, sondern ein Überbleibsel des alten Systems. ${ }^{30}$ Die chinesische Regierung sucht weiterhin nach einem Ausgleich zwischen den Vorteilen einer Dezentralisierung und den Gefahren eines daraus resultierenden Machtverlustes an die Provinzen. In der Reformära sind die lokalen Strukturen gestärkt worden, indem ihnen neue Aufgaben - und damit auch die Verantwortung für mögliche Fehlentwicklungen - übertragen worden sind, sodass das System in mancher Hinsicht de facto föderalistische Strukturen aufweist. An dem zentralistischen Machtanspruch der Partei jedoch darf nicht gerüttelt werden. Es erweist sich als enorm schwierig, das richtige Mischungsverhältnis von Re- und Dezentralisierung zu finden.

Eine Parteiendemokratie steht in der Ordnungsdebatte der KPCh nicht zur Diskussion. Der Zentralismus und die Steuerung durch die Kommunistische Partei sollen nicht aufgegeben werden. Allerdings wird von vielen chinesischen Politikwissenschaftlern der Gedanke einer Stärkung des Parlaments und seines Begleitorgans, der Politischen Konsultativkonferenz des Chinesischen Volkes, vorgeschlagen. Durch den Ausbau deliberativer Strukturen sollen neue indirekte Kanäle der Partizipation eröffnet werden, wodurch die seit vielen Jahren immer eklatanter zutage tretende Distanz zwischen Partei-Staat und Gesellschaft abgebaut werden soll. ${ }^{31}$

28 Ren 2013.

29 Chu 2012.

30 Tocqueville 2013, S. 55. Diskutiert wird dieser Punkt unter anderem von Liu 2001, S. 99.

31 Yang 2013, S. 223.

Leviathan, 42. Jg., 3/2014 
Mit der Ordnungsfrage verbunden ist die Frage nach dem institutionellen Aufbau des Systems und seiner Fähigkeit, auf die Interessen und Forderungen der verschiedenen Bevölkerungsgruppen einzugehen. Ein Wandel innerhalb des (sozialistischen Ein-Parteien-)Systems ist die chinesische Antwort auf diese Frage. Ministerien werden umgebaut, zusammengelegt, Kompetenzen neu verteilt. Auf keinen Fall, so der allgemeine Konsensus, dürfe es zu einer institutionellen Erstarrung des Systems kommen. Denn eine solche, so Yang Zhihuang von der Peking Universität, werde über lange Sicht zwangsläufig zu seiner Degenerierung, wenn nicht gar seinem Zusammenbruch führen. Die Französische Revolution, so ergibt diese Lesart der Schriften Tocquevilles, sei als ein gesellschaftlicher Widerstand gegen den Stillstand und die Verweigerung von Reformen zu lesen. ${ }^{32}$

Analog hierzu wird auch der Niedergang der Sowjetunion (SU) - ein System, das aufgrund seiner Nähe zum chinesischen Modell direktere Parallelsetzungen und Schlussfolgerungen ermöglicht - auf die Hybris der Kommunistischen Partei der SU zurückgeführt, ihre Lehren trotz aller sozio-ökonomischen Krisenerscheinungen unverändert weiter gepredigt zu haben, die Institutionenordnung nicht flexibilisiert und durch die Unfehlbarkeitsformel des sowjetischen Sozialismus jede Form einer systemrettenden Reform kategorisch abgelehnt zu haben. ${ }^{33}$ Schnell wird deutlich, dass dieses Bild eines dogmatisch erstarrten sowjetischen Systems der historischen Realität kaum gerecht wird. Lenins Neue Politische Ökonomie wird hier ebenso ausgeblendet wie Experimente mit einer temporären Lockerung der zentralistischen Planung. Was am sowjetischen Vergleichsmodell bereits auf den ersten Blick erkennbar ist, gilt in ähnlicher Form auch für das französische Referenzmodell: Hier wird ein Anti-Modell konstruiert, gegen das ein idealtypisches »chinesisches « Sozialismusmodell entworfen werden kann.

Die generelle Notwendigkeit von » Reformen «, ein Begriff, der in der chinesischen Debatte als passe partout für die diversen Wünsche und Erwartungen der verschiedensten Interessensgruppen Verwendung findet, ist innerhalb der chinesischen Parteiführung unumstritten. ${ }^{34}$ Bei den durch die politische Führung diskutierten Reformen geht es allerdings weniger um eine politische Liberalisierung als vielmehr um eine Effizienzsteigerung und Professionalisierung der administrativen Strukturen. Uneinigkeit besteht hinsichtlich der konkreten Restrukturierung der Beziehungen zwischen Politik und Ökonomie, zwischen Partei-Staat und Gesellschaft. Exemplarisch verdeutlicht dies das Nachsinnen über das bei Tocqueville thematisierte Zusammenspiel von Freiheit (ziyou) und Gleichheit (pingdeng). Während die ärme-

32 Ebd., S. 214.

33 Pang, Sun 2013, S. 30-31.

34 Vor die Entscheidung Reform oder Revolution gestellt, plädiert die chinesische Führung für Reformen, allerdings nicht ganz unisono. Wen Jiabaos Vorstoß, dass »ökonomische Reformen ohne politische Reformen zum Scheitern verurteilt seien «, mit dem er 2008 einen Topos der frühen 1980er Jahre erneut zum Leben erweckte, wurde und wird nicht von allen Parteimitgliedern unterstützt - im Gegenteil, seine Rede in Shenzhen, in welcher er diesen Gedanken ausformulierte, wurde lediglich in den Lokalmedien abgedruckt. Parteiintern aber wurde infolge der Rede durchaus über das korrekte Verhältnis von Reform und Revolution debattiert. 
ren Einkommensschichten, so die Darstellung der Reflektionen Tocquevilles bei Xia Liping, nach Gleichheit strebten, formulierten die reicheren Schichten ihren Anspruch auf Freiheit. Um der ersteren Gruppe zu entsprechen, wäre eine zentralstaatliche Redistributionspolitik ein mögliches Lösungsmodell, jedoch könnte dies mit einem Verlust an Freiheitsrechten für die reicheren Schichten Hand in Hand gehen. ${ }^{35}$ Eine im Mai 2013 an der Shanghaier Akademie für Sozialwissenschaften durchgeführte Tagung diskutierte in diesem Sinne, gestützt auf Tocqueville, die Überlegung, dass es in Zeiten extremer sozialer Ungleichheiten möglicherweise zum Schutz der Rechte des Proletariats unverzichtbar sei, die allgemeine »Freiheit « (temporär) einzuschränken. ${ }^{36}$

Nicht die Armut, sondern das Streben nach Gleichheit (pingdeng) habe die Menschen in Frankreich, so Yang Zhihuang, gegen das alte System auf die Straße getrieben. Hierbei betont Yang drei Ausprägungen von Ungleichheit im vor-revolutionären Frankreich: Ungleichheiten zwischen den Gesellschaftsschichten, innerhalb der Noblesse sowie auch zwischen urbanen und ländlichen Räumen ${ }^{37}$ - woraus sich indirekt auf die Dilemmata der gegenwärtigen VR China rückschließen lässt. Konkurrenz und Spannungen bestehen dort zwischen den neuen Einkommensgruppen und Bevölkerungsschichten, die sich seit der Einleitung der Reform- und Öffnungspolitik herausgebildet haben. Aber auch innerhalb der ökonomischen Eliten, insbesondere zwischen den Staatssektoren und der neuen Privatwirtschaft, unter den Intellektuellen wie auch zwischen den konkurrierenden Faktionen der Kommunistischen Partei der VR China, werden harte Kämpfe um Macht und Einfluss, Status und Position ausgetragen. Grund für die Kämpfe um die »richtige Linie« sind die extremen Entwicklungsdisparitäten zwischen Stadt und Land, den reichen Küstenprovinzen im Osten und den unterentwickelten Westregionen. Das Heer der Wanderarbeiter, das sein Glück in den weiter wachsenden chinesischen Megametropolen sucht und dort weitgehend informellen Beschäftigungen nachgeht, stellt das politische System vor eine kaum zu bewältigende Steuerungsaufgabe. Das Problem ist das Hukou-System, eine Art Haushaltsregistrierung, welche die Menschen nach ihrem Geburtsort dem städtischen oder aber dem ländlichen Raum zuordnet. Mit dieser Registrierung ist der Zugang zu sozialen Leistungen verbunden. Schränkte das Hukou-System noch in der maoistischen Ära die Mobilität seiner Bevölkerung ein, um der Landflucht entgegenzuwirken, so hat es sich mittlerweile zu einem Instrument der Diskriminierung entwickelt, das die Bevölkerung in zwei Klassen mit unterschiedlichen Privilegien einteilt. Seit vielen Jahren wird über die Notwendigkeit diskutiert, das Hukou-System abzuschaffen, wenigstens aber zu reformieren. In Analogie zu den Auslösern der Französischen Revolution nach Tocqueville lautet hier das indirekte Argument der Reformbefürworter, dass eine erneute, diesmal aber nicht von der Partei geplante Revolution nur abgewendet werden könne, wenn der

\section{Xia 2013, S. 5.}

36 Eine Zusammenfassung der wichtigsten Punkte der Konferenz ist in Guoji Guanxi Yanjiu (Journal of International Relations) (2013/03) abgedruckt worden. Ähnliche Überlegungen finden sich bereits bei Qiao 2009, S. 102.

37 Yang 2013, S. 215.

Leviathan, 42. Jg., 3/2014 
Wunsch der Bevölkerung nach Gleichheit erfüllt werde. Das 3. Plenum des 18. ZK, das sich im November 2013 mit der Formulierung der ökonomischen Entwicklungsstrategie für die neue chinesische Führung beschäftigte, sieht die Lösung der Hukou-Problematik als einen zentralen Punkt des Reformplans an. ${ }^{38}$ Inwieweit dieser aber auch umgesetzt werden kann, bleibt so lange fragwürdig, wie die Zentralregierung daran festhält, die Kosten hierfür allein den Städten zu überlassen (die dann also auch ihre Schulen, die Krankenhäuser und die sozialen Sicherungssysteme für die Wanderarbeiter und ihre Familien öffnen und die entstehenden Kosten aus dem laufenden Haushalt finanzieren müssten).

Die eigentliche Frage, welche die chinesischen Wissenschaftler mit Blick auf Tocqueville beantworten wollen, lautet, wie eine Revolte gegen das System abgewendet werden kann. Die Französische Revolution und ihre Folgejahre werden folglich nicht thematisiert, um die Legitimität der alten Revolution, die zur Gründung der VR China führte, zu untermauern. Bei einer kritischen Lektüre der hierzu erschienenen chinesischen Texte wird schnell deutlich, dass die offiziell aus der Lektüre Tocquevilles gewonnenen Grundaussagen das gegenwärtige China und seine akuten sozio-ökonomischen Herausforderungen reflektieren und das revolutionäre und post-revolutionäre Frankreich des 18. Jahrhunderts als historisches Beispiel für bestimmte gesellschaftliche Bewegungen betrachten, aus denen man lernen kann. So lautet eine zentrale Empfehlung an die chinesische Partei-Regierung, keine öffentliche Meinung gegen das System entstehen zu lassen, sondern durch eine gezielte Medienpolitik die Bevölkerung auf den neuen Slogan des gemeinsamen »Chinesischen Traums « einzuschwören, ${ }^{39}$ den Xi Jinping kurz nach seiner Ernennung zum Generalsekretär der Kommunistischen Partei ins Leben gerufen hatte. Unter dem Mantel des »Chinesischen Traums « erfolgen Debatten über die Neuausrichtung und Reformulierung des Sozialismus mit chinesischen Charakteristika, welche nicht bestehende Missstände legitimieren, sondern ein langfristiges Ideal, eine Art utopische Zielvorgabe fixieren. ${ }^{40}$

Diese Frage nach dem Verlauf des Staatsbildungsprozesses im Nachgang einer Revolution und der Errichtung und Konsolidierung einer neuen Gesellschaftsordnung ist eines der Leitmotive in der Untersuchung Tocquevilles. Damit bieten sich mehrere Interpretation der chinesischen Tocqueville-Debatte an: Es geht möglicherweise nicht nur darum, die reformorientierten Kräfte auf die Gefahren und negati-

38 Communiqué des 3. Plenums des 18. ZK 2013. http://german.china.org.cn/china/ 2014-01/15/content_31202962.htm (Zugriff vom 10.01.2014).

39 Yang 2013, S. 222-223.

40 Dass es sich bei dem »Chinesischen Traum « von einer allumfassenden Harmonie um ein utopisches, also realiter nie erreichbares Ziel handelt, verdeutlich die Verwendung des Begriffs datong in Xi Jinpings Rede, der für die große chinesische Einheit und Gemeinschaft in einem längst verlorenen Goldenen Zeitalter steht. Dieser taucht - ähnlich wie der Begriff des tianxia, der symbolisch für das chinesische Kaiserreich und den chinesischen Herrschaftsanspruch steht - in Texten auf, die einen Gegenentwurf zu den bestehenden Systemstrukturen propagieren - also bei den Qing-Reformern des späten 19. Jahrhunderts ebenso wie bei den gegenwärtigen Verfechtern einer weitergehenden Systemliberalisierung. 
ven Begleiterscheinungen eines revolutionär eingeleiteten Systemwandels hinzuweisen, sondern vielleicht auch darum, die gegenwärtigen Systemauswüchse nicht als Scheitern der Gründungsrevolution der VR China, sondern als schwierige Durchgangsphase zu klassifizieren. Auch nach 1789, wie Tocquevilles Analyse zeigt, bestanden Strukturen des alten Systems weiter fort, kam es zu neuen Fehlentwicklungen und Gewaltexzessen. Liu Chenguang von der Zentralen Parteihochschule hält fest, dass die Revolution in China, die zur Begründung eines neuen Systems geführt habe, bereits erfolgt sei und es folglich nun um weitere Schritte zur Realisierung der ursprünglichen Zielvorgaben gehe, welche durch Reformen erreicht werden sollten. ${ }^{41}$

Auch manche der sich »anonym « zu Wort meldenden chinesischen Internetteilnehmer sehen in Tocquevilles Studie ein Spiegelbild des gegenwärtigen China. Allerdings findet unter diesen durchaus die Idee Zuspruch, dass Tocquevilles Beschreibung der sozio-ökonomischen Spannungen vor dem Ausbruch der Französischen Revolution Ähnlichkeiten mit den gegenwärtigen Entwicklungsproblemen der VR China aufweise - im Unterschied zur oben skizzierten staatstragenden Auslegung, welche diese Missstände als Übergangsphänomen ausweist, gilt hier die »bürgerliche « Revolution als weiterhin noch bevorstehend. ${ }^{42}$

Im Kontext der Tocqueville-Debatte findet zudem eine Typologisierung von legitimen und illegitimen Formen der Revolution statt. Die Xinhai-Revolution, auf die sich auch der Gründungsmythos der Kommunistischen Partei der VR China beruft, steht auf dieser Matrix für den legitimen Widerstand gegen das feudale, imperiale System des chinesischen Kaiserreichs. Erst nach der Vereinnahmung dieser Bewegung durch die Konkurrenzpartei - die chinesischen Nationalisten unter Chiang Kai-shek - gilt diese als gescheiterte beziehungsweise verratene Revolution, deren Ziele erst mit der Etablierung der VR China verwirklicht wurden.

Referenzmodell für die Frage nach Reform versus Revolution wiederum ist die späte Qing-Dynastie. Als Reaktion auf die Opiumkriege war zunächst eine Selbststärkungsbewegung mit dem Ziel der Restauration der alten Ordnung unter dem Motto »die chinesischen Wissenschaften als Essenz, die westlichen Wissenschaften für die praktische Anwendung « eingeleitet worden. China übernahm die Technologien des Westens, hielt aber an seiner eigenen Staats- und Gesellschaftskonzeption fest. Spätestens mit der Niederlage Chinas gegen Japan 1894/1895 wurde diese Restaurationsbewegung jedoch als gescheitert eingestuft. Es folgte ein Ideenwettkampf unter den verschiedenen intellektuellen Gruppierungen, wie das Überleben Chinas als souveräner Staat (und dem eigenen Anspruch nach als Kaiserreich) in Anbetracht der zunehmenden Fremdkontrolle gewährleistet und die Selbstbestimmung wiederhergestellt werden könnte. War zunächst der Konfuzianismus als

41 Liu 2013.

42 Eine Auswahl von übersetzten Tocqueville-Kommentaren auf chinesischen Mikroblogs findet sich unter http://globalvoicesonline.org/2012/12/28/tocqueville-french-revolution-classic-on-chinas-bestsellers-list/ (Zugriff vom 02.07.2014).

Leviathan, 42. Jg., 3/2014 
reformblockierendes Element eingestuft worden, formulierte Kang Youwei ${ }^{43}$ Ende des 19. Jahrhunderts die These, dass Konfuzius durchaus als Reformer seiner Zeit gesehen werden könne und nur seine Lehren über die Jahre hinweg fehlinterpretiert und verfälscht worden seien. Andere wiederum traten für anarchistische Ideen, wieder andere für eine komplette Verwestlichung des Systems ein. ${ }^{44}$ Nachdem 1898 die Hundert-Tage-Revolution, die eine konstitutionelle Monarchie anstrebte, zunächst durch den Kaiserhof niedergeschlagen worden war, war es der Kaiserhof selbst, der zu Beginn des 20. Jahrhunderts den Auftrag zur Formulierung einer chinesischen Verfassung und zu umfassenden Reformen des Beamtenapparats gab. Der Ausbruch der Xinhai-Revolution 1911, welche das dynastische System zerstörte, wird in der chinesischen Debatte über Reform und Revolution als Beleg dafür zitiert, dass eine zu zögerliche Umsetzung oder Blockierung von Reformen nicht den Fortbestand der alten Ordnung zementiere, sondern den Untergang noch zusätzlich beschleunige.

Das Horrorszenario, das in den Köpfen der politischen Führung weiterhin spukt, findet sich in Tocquevilles Feststellung, dass die Einführung von Reformmaßnahmen für eine Regierung die gefährlichste Phase mit Blick auf ihren Autoritätserhalt darstelle. Schwere Zeiten würden im Normalfall keine Revolten gegen das System hervorrufen - beziehungsweise erst dann, wenn jemand behaupten würde, eine Lösung der Probleme parat zu haben. Denn dann wären die Krisen in den Augen der Bevölkerung nicht länger ein unabänderbares Übel, sondern ein Missstand, der sich qua politischer Aktionen beheben ließe. ${ }^{45}$

Mit den chinesischen Debatten zu Tocquevilles Schriften zur Revolution finden wir eine Fortschreibung der marxistischen Grundannahmen, die in der maoistischen Ära die Diskurse geprägt hatten und bis heute fortwirken. In der gegenwärtigen chinesischen politischen Philosophie wird das marxistische Revolutionskonzept als eine Revolution der Gesellschaft verstanden, die durch ökonomische Faktoren hervorgerufen wird und an deren Ende das Ziel der Gleichheit (pingdeng) stehen soll. Tocquevilles Revolutionsbegriff hingegen wird verstanden, als ob er vor allem auf die Erlangung der Freiheit (ziyou) abziele. ${ }^{46}$ Es scheint, dass in der gegenwärtigen politischen Debatte diese beiden Ansätze nun integriert gedacht werden. An die Stelle der maoistischen Vorstellung einer " permanenten Revolution " sind nun Überlegungen zu neuen Steuerungs-Formen getreten, welche neue Dimensionen der Partizipation via Deliberation umfassen und die Rechte und Forderungen der verschiedenen gesellschaftlichen Gruppen stärker einbinden sollen - und damit parallel Gleichheit und Freiheit (jedoch nicht Demokratie) anstreben.

Der Rückgriff auf die europäische Staatsphilosophie ist allerdings kein Alleinstellungsmerkmal der fünften chinesischen Führungsgeneration um Xi Jinping und Li Keqiang. Im Bücherregal des vorherigen chinesischen Ministerpräsidenten, Wen

43 Kang Youwei (1858-1927): politischer Denker der späten Kaiserzeit, Ideengeber der 100Tage-Reformen des Jahres 1898.

$44 \mathrm{Zu}$ den konkurrierenden Intellektuellengruppierungen und ihren Ideen vgl. Chang 1980.

45 Vgl. Zhu 2013, S. 28.

46 Sui, Zhang 2013, S. 13. 
Jiabao, standen Marc Aurels Meditationen. ${ }^{47}$ In diesen geht es weit weniger um die Frage nach Revolution versus Reform, sondern allgemein um den moralisch richtigen Führungsstil, das Verhältnis von Herrschenden und Beherrschten. Entsprechende Betrachtungen finden sich auch in der chinesischen Staatsphilosophie, die in den letzten Jahren reaktiviert und gezielt in den politischen Diskurs eingebettet worden ist. So widmen sich ganze Forschungsprojekte an den chinesischen Eliteuniversitäten der Aufarbeitung der staatsphilosophischen Schriften aus der Zeit vor der ersten chinesischen Reichseinigung (221 v. Chr.), als sich die »Hundert (Philosophen-)Schulen « einen produktiven Ideenwettstreit lieferten. Der aus diesem Wettstreit siegreich hervorgegangene Konfuzianismus diente schließlich über viele Jahrhunderte nicht nur als Staatsphilosophie, sondern auch bis zum Ende des dynastischen Systems als Staatsdoktrin und legitimatorische Grundlage des Mandarinats. Erst mit den ikonoklastischen Bewegungen des frühen 20. Jahrhunderts und dem Siegeszug des Marxismus fielen diese Ideen offiziell in Ungnade. In den letzten Dekaden ist jedoch kein Mono-Marxismus entstanden, sondern eine Sinisierung und Synthese von importierten (marxistischen) Grundideen und Kernelementen der vormodernen Staatsphilosophie (Stichworte: Harmonische (sozialistische) Gesellschaft; Harmonische Welt). Unter der vierten Führungsgeneration (Hu Jintao, Wen Jiabao) sind diese traditionellen staatsphilosophischen Ideenkonzepte (und ihre neologistischen Weiterschreibungen) schließlich rehabilitiert worden und folglich auch akademisch wieder salonfähig geworden. ${ }^{48}$

Welche Quellen - chinesische oder westliche Schlüsseltexte - gelten als Wegweiser für die weiteren Reformschritte? Stark vereinfachend lässt sich festhalten, dass im Bereich der ideellen und symbolischen Herrschaftslegitimierung ein Rückgriff auf wiedergefundene beziehungsweise erfundene philosophische Traditionen und Ideenströmungen stattgefunden hat. Mit Blick auf konkrete Lösungsmodelle wiederum sind es auch die Lehren aus historischen Erfahrungen und Entwicklungsmustern in anderen Staaten und Regionen, welche den Rezeptions- und Adaptionsprozess prägen. Die Tocqueville-Rezeption weist sowohl Bezüge zu der abstrakten symbolischen Debatte wie auch zu der Suche nach Lösungsmodellen auf. Konkrete Lehren für China werden aus Tocquevilles Studie jedoch eher selten abgeleitet, vielmehr werden in Anlehnung an sein Werk grundlegende historische Entwicklungsmechanismen im Nach- und Vorfeld von Revolutionen debattiert. Mit dem Umweg über Frankreich - und mit dem symbolischen Abstand von über 200 Jahren zur Gegenwart - lassen sich in China Diskussionen über Themen führen, die sonst möglicherweise als destabilisierend eingeordnet werden könnten. Es handelt sich dabei um Spiegeldebatten, bei denen nicht die wissenschaftliche Aufarbeitung der historischen Hintergründe, sondern die Erarbeitung einer neuen Entwicklungsstrategie für China im Mittelpunkt steht und andere Systeme als Vor-, Gegen- oder Zerrbilder des chinesischen Modells herangezogen werden.

47 Vgl. den Artikel »Book preferences hint at China's will for mild reform «, in China Daily vom 8. Januar 2012. www.chinadaily.com.cn/life/2012-11/08/content_15895481.htm (Zugriff vom 10.01.2013).

48 Bell 2008.

Leviathan, 42. Jg., 3/2014 


\section{Literatur}

Bell, Daniel A. 2008. China's new confucianism. Princeton: Princeton University Press.

CASS Institut für Marxismus-Studien 2011. »Biange zhong de shehuizhuyi si guo « (Vier sozialistische Staaten im Wandel), in Dangdai Shijie yu Shehuizhuyi (Contemporary World \& Socialism) 5, S. 44-50.

Chang, Hao 1980. "Intellectual change and the reform movement, 1890-8 «, in The Cambridge History of China, Band 11, hrsg. v. Fairbank, John K., S. 274-338. Cambridge: Cambridge University Press.

Chu, Jianguo 2012. Faguo da geming yin he chengxiao bu da (Warum die Französische Revolution $\mathrm{zu}$ keinen großen Resultaten führte). http://book.ifeng.com/gundong/ detail_2012_12/25/20490835_0.shtml (Zugriff vom 20.05.2014).

Dickson, Bruce J. 2003. Red capitalists in China: the party, private entrepreneurs, and prospects for political change. New York: Cambridge University Press.

Dobbs, Michael 1989. House of cards. London: Collins.

Erber, Georg. 2014. »China: Land der unbegrenzten Möglichkeiten? «, in Leviathan 42, 1, S. 29-66.

Hu, Deping. 2013. Pojie » Jiu zhidu yu da geming « zhi wen (Erläuterung zu einigen Fragen in »Der alte Staat und die Revolution«). www.21ccom.net/articles/read/article_2013081089529.ht $\mathrm{ml}$ (Zugriff vom 25.01.2014).

Jiang, Shixue 2010. "Zhongguo tese shehuizhuyi yu Guba tese shehuizhuyi zhi bijiao « (Vergleich der Sozialismen mit chinesischen und mit kubanischen Charakteristika), in Zhongguo Tese Shehuizhuyi Yanjiu (Studies on Socialism with Chinese characteristics) 2, S. 31-37.

Liao, Rebecca 2013. "Tocqueville in China ", in Dissent: A Quarterly of Politics and Culture. www.dissentmagazine.org/online_articles/tocqueville-in-china (Zugriff vom 10.04.2014).

Lilla, Mark 2011. "Leo Strauss in Peking ", in Leviathan 39, 2, S. 195-200.

Liu, Beicheng 2001. »Tuokewei'er guanyu Faguo da geming qiyin de jieshi« (Tocquevilles Erklärung zu den Entstehungsgründen der Französischen Revolution), in Beijing Shifan Daxue Xuebao (Journal of Beijing Normal University) 2, S. 97-102.

Liu, Chenguang 2013. Jiu zhidu yu da geming de wudu yu landu (Fehlerhafte und überinterpretative Lesungen von »Der alte Staat und die Revolution «). http://history.sina.com.cn/his/zl/ 2013-08-21/205551764.shtml (Zugriff vom 10.01.2014).

Nathan, Andrew 2003. "Authoritarian resilience", in Journal of Democracy 1, S. 6-17.

Noesselt, Nele 2014. "Staatlich-zivile Interaktionsmuster im Wandel: Governance-Konzepte der neuen chinesischen Führungselite ", in Modernes Regieren in China, hrsg. v. Heinelt, Hubert, S. 137-157. Baden-Baden: Nomos.

Pang, Bo; Sun, Dinghe 2013. »Sulian jieti de yuanyin ji jiaoxun zai fenxi« (Neue Analyseansätze zum Niedergang der Sowjetunion und den Lehren [für China]), in Theory Research 2, S. 30-31.

Qiao, Guiping 2009. "Minzhu yu ziyou de chongtu yu pingheng (Konflikt und Ausgleich zwischen Demokratie und Freiheit), in Hunan Xingzheng Xueyuan Xuebao (Journal of Hunan Administration Institute) 4, S. 102-105.

Ren, Junfeng 2013. Tuokeweirer yu Zhongguo (Tocqueville und China). http://news.sina.com.cn/ $\mathrm{pl} / 2013-07-19 / 095927714025$.shtml (Zugriff vom 25.03.2014).

Sandel, Michael J. Hrsg. 2007. Justice. Oxford: Oxford University Press.

Senghaas, Dieter 1996. »Wie geht es mit China weiter? «, in Leviathan 24, 1, S. 78-92.

Shambaugh, David 2008. »Learning from abroad to reinvent itself: external influences on internal CCP reforms", in China's changing political landscape: prospects for democracy, hrsg. v. Cheng, Li, S. 283-301. Washington: Brookings.

Sui, Hongbo; Zhang, Dun 2013. "Makesi he Tuokewei'er zhengzhi zhexue zhong de geming gainian " (Der Revolutionsbegriff in der politischen Philosophie von Marx und Tocqueville), in Journal of Party School of Tianjin Committee of the CPC 6, S. 13-18.

Tocqueville, Alexis de 1954 [1893]. Erinnerungen. Stuttgart: Deutsche Verlags-Anstalt.

Tocqueville, Alexis de 2013 [1856]. Der alte Staat und die Revolution. Warendorf: Johannes G. Hoof (Bibliothek des skeptischen Denkens).

Tong, Yanqi; Lei, Shaohua 2010. »Large-scale mass incidents and government responses in China ", in International Journal of China Studies 1, S. 487-508. 
Wang, Yuewen 2010. Da Qing xiangguo (Der Minister der Qing-Dynastie). Shijiazhuang: Huashan Wenyi Press.

Xia, Liping 2013. »Ziyou yu pingdeng guanxi xiyao: Yi ge Tuokewei’er mingti (Zu den Beziehungen zwischen Freiheit und Gleichheit: Zu einer These von Tocqueville), in Shandong Social Sciences 12, S. 5-9.

Xinhua 2013. Xi Jinping Vows »Power within Cage of Regulations « [22.01.2013]. http:// news.xinhuanet.com/english/china/2013-01/22/c_132120363.htm (Zugriff vom 24.01.2013).

Yang, Zhihuang 2013. »Tizhi jiangting, tizhi fenghua, yu tizhi nianxing: Du Wang Qishan jian shu jian lun Faguo da geming dui Zhongguo de qishi « (Erstarrung, Degenerierung und Kohäsionskraft des institutionellen Systems: Zu dem von Wang Qishan empfohlenem Buch »Der alte Staat und die Revolution « und den Lehren der Französischen Revolution für China), in Comparative Economic and Social Systems 6, S. 214-225.

Zhu, Zhenglin 2013. "Gaige de fengxian yu huajie" (Die Gefahren der Revolution und ihre Auflösung), in Dushu 11, S. 28-35. 
Zusammenfassung: Tocquevilles Werk Der alte Staat und die Revolution gilt als Schlüssellektüre der obersten chinesischen Führungselite. Dies überrascht, denn die Forderung nach Demokratie und Menschenrechten scheint dem politischen System der VR China geradezu diametral entgegenzustehen. Anknüpfungspunkt der Debatte ist jedoch nicht die Demokratisierungsthematik, sondern das Nachsinnen über Revolution, Reform und die Konsolidierung politischer Herrschaft.

Stichworte: Alexis de Tocqueville, China, Legitimität, Reform, Revolution

\section{Alexis de Tocqueville in China: mirror debates on the need for reform and the threat of revolution}

Summary: Tocqueville's study The old regime and the revolution is required reading for China's political elite. This appears to be paradoxical, since demands for democracy and human rights are diametrically opposed to China's political system. The starting point of the debate is not issues of democratization, however, but the reflections on revolution, reform and the consolidation of political rule.

Keywords: Alexis de Tocqueville, China, legitimacy, reform, revolution

\section{Autorin}

Dr. Dr. Nele Noesselt

c/o GIGA Institut für Asienstudien

Rothenbaumchaussee 32

20148 Hamburg

$040 / 42887446$ 